bioRxiv preprint doi: https://doi.org/10.1101/2021.05.17.444398; this version posted May 17, 2021. The copyright holder for this preprint (which

was not certified by peer review) is the author/funder, who has granted bioRxiv a license to display the preprint in perpetuity. It is made available under aCC-BY-NC-ND 4.0 International license.

Probabilistically Weighted Multilayer Networks disclose the link between default mode network instability and psychosis-like experiences in healthy adults

\title{
Probabilistically Weighted Multilayer Networks disclose the link between default mode network instability and psychosis-like experiences in healthy adults
}

\author{
Simone Di Plinio ${ }^{1, *}$, Sjoerd J H Ebisch ${ }^{1,2}$
}

1 Department of Neuroscience, Imaging, and Clinical Sciences, G D'Annunzio University of ChietiPescara, Chieti, Italy

2 Institute for Advanced Biomedical Technologies (ITAB), G D'Annunzio University of ChietiPescara, Chieti, Italy

*Corresponding author: simone.diplinio@unich.it

Short title: Probabilistically Weighted Multilayer Networks

Keywords: Multilayer networks; Dynamic connectivity; Default mode network; Psychotic experiences;

Functional networks; Flexibility 
Probabilistically Weighted Multilayer Networks disclose the link between default mode network instability and psychosis-like experiences in healthy adults

\begin{abstract}
The brain is a complex, multilayer system in which the functional interactions among its subunits vary over time. The trajectories of this dynamic variation contribute to interindividual behavioral differences and psychopathologic phenotypes. Despite many methodological advancements, the study of dynamic brain networks still relies on biased assumptions in the temporal domain. The current paper has two goals. First, we present a novel method to study multilayer networks by modelling intra-nodal connections in a probabilistic and biologically driven way. We introduce a temporal resolution of the multilayer network based on signal similarity within time series. This new method is tested on synthetic networks by varying the number of modules and the sources of noise in the simulation. Secondly, we implement these probabilistically weighted (PW) multilayer networks to study the association between network dynamics and subclinical, psychosisrelevant personality traits in healthy adults. We show that the PW method for multilayer networks outperform the standard procedure in modular detection and is less affected by increasing noise levels. Additionally, the PW method highlighted associations between the temporal instability of default mode network connections and psychosis-like experiences in healthy adults. PW multilayer networks allow an unbiased study of dynamic brain functioning and its behavioral correlates.
\end{abstract}


Probabilistically Weighted Multilayer Networks disclose the link between default mode network instability and psychosis-like experiences in healthy adults

\section{INTRODUCTION}

Multilayer networks rely on the assumption that different network layers can be combined in supramodal entities given that elements (nodes) occupying the same place in each network may be considered as naturally linked across time or across experimental conditions of a $\operatorname{task}^{1-5}$. Defining a supramodal structure allows to investigate dynamic changes in a network indexed by dynamic nodal measures such as promiscuity, flexibility, integration, and recruitment $^{6-9}$. Multilayer networks have increasingly been employed in both basic and clinical neuroscience, and allowed to describe interesting patterns of dynamic network rearrangement underlying normal behavior ${ }^{10,11}$ as well as clinical conditions such as depression ${ }^{12}$ and schizophrenia ${ }^{13}$.

However, actual models of multilayer brain networks are lacking an objective methodological procedure to weight network parameters in a biologically-sound fashion. The topological and modular properties of multilayer brain networks rely on two key parameters, namely gamma $(\gamma)$ and omega $(\omega)$. The former, $\gamma$, indicates the weight of the null models when computing modularity ${ }^{14-16}$. Thus, increasing the value of $\gamma$ results in a more fragmented network structure with many small modules ${ }^{17-20}$. The latter, $\omega$, represents the empirical strength of connections that a node has toward himself in consecutive (if multilayer networks are built across time) or alternative (if multilayer networks are built across experimental conditions) time intervals ${ }^{21,22}$, so that $\omega$ essentially controls the intra-layer versus cross-layer modular architecture. The actual standard is to set $\omega$ to a fixed number (usually $\omega=1$ ) but, as also noted by many scientists in the field, such assumption is unmotivated and introduces strong biases in the analysis.

Thus, the commonly used approach implies arbitrariness regarding the values of the detection of the modular architecture. In particular, by setting $\omega=1$, dynamic (temporal) oscillations of nodal activity over time are non-efficiently integrated in the cross-layer 
Probabilistically Weighted Multilayer Networks disclose the link between default mode network instability and psychosis-like experiences in healthy adults

modular detection ${ }^{23,16}$. Many studies that investigated dynamic evolutions of brain activity and functional connectivity highlighted classes of brain regions with peculiar functional properties in the time domain ${ }^{24-26}$. For example, regions in the inferior parietal lobule, medial premotor cortex, and posterior cingulate cortex have been labelled as functional hubs ${ }^{27}$ since they dynamically link regions in their own modules (cingulo-opercular network, sensorimotor network, and default mode network, respectively) with other brain modules ${ }^{28,29}$. The efficient integration of information across networks is crucial both for the control of explicit, externally-directed actions ${ }^{30,31}$ and for implicit, self-related information processing ${ }^{32}$. Aberrant connectional profiles, especially in regions related to the default mode network, seem to underlie many psychopathological conditions ${ }^{33,34}$, and these characteristics may depend on dysfunctional dynamic processes ${ }^{10,35,36}$. Thus, the implementation of a degree of control over the temporal domain is necessary to increase the ability to detect true transient module configurations during conscious experiences, which are naturally occurring both during task execution and during task-free states ${ }^{37}$.

Here, we introduce a further parametrization of the detection of dynamic modular architectures with the aim to improve the biological appropriateness of community detection in multilayer networks. As described above, the current estimation of the modularity relies on a structural $(\gamma)$ and on a pseudo-structural $(\omega)$ parameter. Instead, the parametrization proposed here accounts or uncertainty in the temporal domain, and replaces $\omega$. We introduce beta $(\beta)$, a parameter that regulates the probability distributions of the strength of edges connecting nodes across multiple layers of a multilayer network (intra-nodal, cross-layer connections). We also implement a biologically-driven way to assign strengths of intra-nodal (temporal) connections from these probability distributions by employing spectral coherence $^{38,39}$ to choose the appropriate probabilistic intra-nodal weights. In other words, our 
Probabilistically Weighted Multilayer Networks disclose the link between default mode network instability and psychosis-like experiences in healthy adults

method probabilistically regulates intra-nodal weights across layers based on biological, temporal properties of the node's signal.

Our approach allows different strengths of cross-layer connections to improve the appropriateness of the multilayer modular detection. The procedure mirrors the current methodology for selecting structural resolutions through the parameter $\gamma$ and aims at investigating modular architectures of the brain by using multiple temporal resolutions as well as multiple structural resolutions, to implement a totally unbiased methodology. The probabilistic multilayer community detection proposed in this paper is expected to increase the sensitivity toward the detection of physiologically plausible, dynamic modular structures, by eliminating the biases constrained by a fixed and "guessed" value of $\omega$, thus allowing for uncertainties over temporal multilayer network features in the same way as $\gamma$ allows the control over spatial network features.

We test this new approach both on simulated data and on real data from resting-state fMRI acquisitions of 39 healthy participants. The performance of the new method was tested together with the standard $\omega=1$ method on synthetic weighted networks obtained by varying the number of modules as well as the weight of structured and unstructured noise in the time series. Moreover, the association between multilayer network properties and individual differences in behavior was tested, focusing on the neural correlates of psychosis-relevant personality traits, which have been hypothesized to be related to self-dysfunction and network segregation ${ }^{32,40,41}$. In particular, subclinical, psychosis-relevant personality traits were evaluated, which can be detected on a continuum in the general population below any threshold of a clinical diagnosis ${ }^{42}$. Such traits can be considered critical from a clinical point of view ${ }^{43}$, as well as from a phenomenological perspective to provide insight into the relationship between neurobiology and the sense of self ${ }^{44}$. 
Probabilistically Weighted Multilayer Networks disclose the link between default mode network instability and psychosis-like experiences in healthy adults

\section{METHODS}

\section{Synthetic Networks}

Three modules in a network of 100 nodes were simulated as follows in 10 consecutive time windows. Nodes were initially assigned to the same module across all the time windows ( $30 \%$ of the nodes were assigned to module A, $50 \%$ to module B, $20 \%$ to module C). Then, $40 \%$ of the nodes were chosen at random to be fast/slow/random oscillators $(15 \%, 15 \%, 10 \%$ respectively) to simulate diverse physiological properties of brain hubs. Fast/slow/random oscillators switched between their module and another random module every 5/3/1 time windows, respectively.

Once defined the modular time-varying structure, time series were created as follows. First, for each time window, a prototype matrix of synthetic functional connections was simulated as a 100x100 symmetric Lehmer matrix. Since in the Lehmer matrix the values of the functional connections increase with proximity to the diagonal and with progressive node numbers, such matrix allows the generation of modules with different degrees of intrinsic connections, thus, avoiding bias related to connection strengths. A time series was simulated for each node from the starting correlation structure and, for each module, the nodes' time series were selectively time-shifted to create modular structures.

Within each node, the time series of 100 voxels were simulated to simulate realistic voxels within a parcel. Initially, for each node, the node's time series was assigned to each voxel within the node. Then, two sources of noise were simulated to represent physiologicallyunrelated noise (unstructured noise) and the "interference" of nearby biological units in the node signal (structured noise). Unstructured noise was simulated - for each voxel - as a random time series that was added to the voxel signal in a weighted manner. Structured noise 
Probabilistically Weighted Multilayer Networks disclose the link between default mode network instability and psychosis-like experiences in healthy adults

was simulated by initially choosing a random [2 to 5] number of attractors on the external surface of the node. A random time series was simulated for each attractor. Then, each voxel in the node suffered each attractor's interference depending on its closeness to the attractor. Finally, to mirror common procedures in neuroimaging, each node time series was obtained by averaging the time series of the voxels within it. Different weights were considered for the two noises (low, medium, high) to investigate the impact of the signal-to-noise ratio to the module detection across the two methods.

Once noisy time series were synthesized, the generalized Louvain function was used to detect modules across time points (http://netwiki.amath.unc.edu/GenLouvain/GenLouvain). The function was implemented both in the "standard" form $(\omega=1)$ as well as using the probabilistically weighted method (PW). The whole procedure was repeated for 100 independent cycles so that, in each cycle, a new modular structure was randomly created. The three levels of structured/unstructured noises were introduced. Other values controlling the procedure, such as $\gamma$ and tau $(\tau)$, were set to default values (1) for simplicity. The weighted approach is described in the next paragraphs. The procedure for obtaining synthetic networks is schematised in Figure 1. To assess the independence of methods' performances on the number of modules in the network, the whole procedure was repeated using five isomorphic modules of equal size ( $20 \%$ of the nodes assigned to each module).

\section{Probabilistically Weighted (PW) Multilayer networks}

To implement control over nodal temporal features of the network, we introduced a matrix of probability weights $\mathrm{W}$. Values in $\mathrm{W}$ were controlled by the temporal resolution parameter $\beta$ and by the temporal features of the nodes. As probability weights, values in $\mathrm{W}$ basically represent the probability of nodes to be self-connected among different layers of the network. Thus, with temporal multilayer networks, $\mathrm{W}$ is a $\mathrm{N}$ by $\mathrm{T}-1$ matrix in which $\mathrm{T}$ is the number of time windows and $\mathrm{N}$ is the number of nodes in the network. By contrast, if working with task 
Probabilistically Weighted Multilayer Networks disclose the link between default mode network instability and psychosis-like experiences in healthy adults

multilayer networks, the matrix $\mathrm{W}$ would be a $\mathrm{K}$ by $\mathrm{N}$ matrix in which $\mathrm{K}$ represents withinnode cross-condition interconnections and is calculated as $\mathrm{K}=\mathrm{Nc}$ ! In which $\mathrm{Nc}$ is the number of experimental conditions (e.g., in a classic two by two factorial psychology paradigm, there would be four conditions, and $\mathrm{K}=4 !=24)$.

To control the strength of cross-layer connections and to mirror the weight of the null model for the structural resolution parameter, values of $\mathrm{W}$ were defined using a probabilistic approach. Initially, a set of Levy alpha-stable distributions, $\Omega$, was generated. Stable distributions rely on four parameters, allowing complete control over the shape and the scale of the distribution. To reflect correlation (functional connectivity), the values of $\mathrm{W}$ (probabilities) were sampled within the interval [0 1]. Thus, the location parameter of the distributions, $\mu$, was set to 0.50 and the scale parameter, $\sigma$, was set to 0.75 . The first shape parameter, $\alpha$, was empirically set to 0.40 , while the second shape parameter, $\beta$, was modulated within the range [-1 1] to obtain a series of distributions in which the head and the tails are arranged progressively in the interval [0 1]. In other words, a set of probability distributions $(\Omega)$ was created in which the ratio of low versus high probabilities was controlled through the parameter $\beta$ : with higher, positive values of $\beta$, the head and the fat tail of a specific distribution in $\Omega$ are located toward lower probability values (lower probabilities of cross-layer connections); instead, with lower, negative values of $\beta$, the head and the fat tail of a specific distribution in $\Omega$ are located toward higher probability values (higher probabilities of cross-layer connections). Values used for $\beta$ in this study were: $[-.75-.250 .25$ $.75]$.

Then, the distributions in $\Omega$ were used to generate values in $\mathrm{W}$ based on the coherence between time intervals. More specifically, we used spectral coherence, also known as magnitude-squared coherence ${ }^{38,39}$, across consecutive time windows. Since coherence estimates the consistency of amplitude and phase between signals across frequencies, it is 
Probabilistically Weighted Multilayer Networks disclose the link between default mode network instability and psychosis-like experiences in healthy adults

biologically appropriate for a node exhibiting coherent signal fluctuations in two adjacent time windows (or in two experimental conditions) $i$ and $j$ to have higher probability to be connected - to be "self-similar" - between the layers $i$ and $j$ (i.e., it occupied the right tail in the stable distribution). Using this procedure, the temporal resolution, $\beta$ (the $\beta$ shape parameter of the stable distributions), regulated the probabilities of connections among adjacent time windows in a way driven by the similarity of the node's signals in the two time intervals. The procedure for estimating probabilistically weighted multilayer modules is illustrated in Figure 2.

The performance of the two methods was assessed by calculating the degree of similarity between the true simulated modular structure and the estimated modular structure through two commonly used indexes for calculating correlations across modular structures, namely the adjusted mutual information index (AMI) and the Rand coefficient.

\section{Application on resting-state data}

The procedure described above for detecting PW multilayer networks, together with the standard $\omega=1$ procedure was implemented also on resting-state data from a sample of 39 healthy participants (19 females and 20 males, aged $23 \pm 2 ; 35$ right-handed and 4 lefthanded) without a history of psychiatric or neurological disease and contraindications for MRI scanning participated in the experiment. The experiment was approved by the local ethics committee. All participants had a normal or corrected-to-normal vision and provided written informed consent before taking part in the study in accordance with the Declaration of Helsinki (2013).

\section{Resting-state Data acquisition}

Each participant performed two consecutive task-free fMRI runs, each consisting of 376 volumes. The participants were instructed to watch a white fixation cross on a black screen without performing a cognitive task. Each run lasted approximately 7.5 minutes. Functional 
Probabilistically Weighted Multilayer Networks disclose the link between default mode network instability and psychosis-like experiences in healthy adults

images were acquired using a Philips Achieva 3T scanner installed at the Institute for Advanced Biomedical Technologies (Gabriele d'Annunzio University, Chieti-Pescara, Italy). Whole-brain functional images were acquired with a gradient echo-planar sequence using the following parameters: repetition time $(\mathrm{TR})=1.2 \mathrm{~s}$, echo time $(\mathrm{TE})=30 \mathrm{~ms}$, field of view $=$ 240x240x142.5 mm, flip angle $=65^{\circ}$, in-plane voxel size $=2.5 \mathrm{~mm}^{2}$, slice thickness $=2.5$ mm. A high-resolution T1-weighted whole-brain image was also acquired after functional sessions using the following parameters: $\mathrm{TR}=8 \mathrm{~ms}, \mathrm{TE}=3.7, \mathrm{FoV}=256 \times 256 \times 180 \mathrm{~mm}$, flip angle $=8^{\circ}$, in-plane voxel size $=1 \mathrm{~mm}^{2}$, slice thickness $=1 \mathrm{~mm}$.

\section{Resting-state Data analysis}

Functional connectivity was calculated as the correlation among average parcels' timeseries for a total of 418 nodes using cortical and subcortical atlases from Joliot and colleagues ${ }^{45}$ plus the cerebellar atlas from Diedrichsen and colleagues ${ }^{46}$. These atlases were chosen because of the integrative method used to define both cortical and subcortical parcels without lateralization biases. The PW method and the standard $\omega=1$ approach were both implemented to detect time-varying modular structures across 10 consecutive time windows of equal length.

We tested if brain-behavioral resting-state associations were differentially detected by the standard $\omega=1$ and by the PW method. Thus, nodal metrices of flexibility, recruitment, integration, and promiscuity were estimated for each subject using both the $\omega=1$ and the PW methods. The behavioral variables were represented by five latent, orthogonal psychosisrelevant factors that have also been used in previous works to study the sense of self and the sense of agency ${ }^{32,47,48}$. We investigated the relation between dynamic multilayer metrics and psychosis-related personality traits with a focus on factors related to psychotic disorders ${ }^{49,50}$. These five factors were reliably (Tucker congruence coefficient $=0.95$ ) derived from a factor analysis with orthogonal (orthomax) rotation on a series questionnaires that were 
Probabilistically Weighted Multilayer Networks disclose the link between default mode network instability and psychosis-like experiences in healthy adults

administered to a larger sample $(\mathrm{N}=101)$ including the present fMRI sample (see references $^{32,47,48}$ for the complete procedure) The questionnaires measured basic as well as psychosis-relevant personality traits: the Big-Five Questionnaire (BFQ short version ${ }^{51}$ ), the tolerance of uncertainty scale (IUS-12 $2^{52}$ ), the Schizotypal Personality Questionnaire (SPQ ${ }^{53}$ ), the Community Assessment of Psychic Experience $\left(\mathrm{CAPE}^{54}\right)$, and the State-Trait Anxiety Inventory $\left(\mathrm{STAI}_{2}{ }^{55}\right)$. The five factors showed the highest loadings in the following subscales: factor 1) STAI2, IUS-inhibitory, CAPE-depression, BFI-neuroticism (negative affect); factor 2) SPQ-cognitive perceptual, CAPE-positive, CAPE-negative (psychosis-like experiences); factor 3) IUS-prospective, BFI-extraversion, BFI-agreeableness, BFI-openness (sociality); factor 4) SPQ-interpersonal, SPQ-disorganizational (schizotypal); factor 5) BFIconscientiousness.

Brain-behavioral relationships were investigated using whole brain, univariate mixedeffects models in which the five psychometric factor scores were used as continuous predictors for each multilayer metric of interest; random intercepts and slopes were added at the individual node level to allow for ROI-specific weighting of brain-behavior correlations; random slopes were added at the subject level. When significant associations were detected, we located the brain structural correlates using best linear unbiased predictors (BLUPs) to generate nodal conditional expectation (ICE) plots and to highlight nodes with the highest contribution.

\section{RESULTS}

Results indicated that the PW multilayer networks outperformed by far the standard $\omega=1$ method in detecting true synthetic multilayer networks. The performance achieved with PW multilayer networks on synthetic data was better with every combination of structured/unstructured noise tested. 
Probabilistically Weighted Multilayer Networks disclose the link between default mode network instability and psychosis-like experiences in healthy adults

With respect to the three-modules structure, the performance of the two methods are reported in Figure 3a-c as indexed by the adjusted information index (AMI) and the Rand coefficient. To note, the performance of the PW method was better than the performance of the standard $\omega=1$ method independently of the $\beta$ value used for calculating weights. P-values for direct t-tests between the performances of the two methods were always smaller than .00001 for both AMI indices and Rand coefficients, confirming a higher performance for the PW method independently of the index or the $\beta$ used. However, as reported in Figure 3, the difference between the performances of the two methods increased with increasing level of noise in the dataset, showing that the PW method is not only more efficient but also less affected by high levels of structured or unstructured noise. The same results were obtained with five modules (Figure 3d-f): the performance of the PW method was still better than the performance of the standard $\omega=1$ method independently of the index or the $\beta$ value used for calculating weights and independently of the noise level (all p-values for direct t-tests < .00001). As it can be noticed comparing standard versus PW methods in Figure 3c and Figure 3f, the standard method did not efficiently detect oscillator nodes and was eventually "confused" by flexible nodes, leading to a worse reconstruction of the true modular structure.

When applied to real data, the PW method was able to identify significant associations between two dynamic metrics, namely flexibility and recruitment, and behavioral measures. Instead, the standard $\omega=1$ method did not identify any significant brain-behavior association. In particular, significant, positive associations were detected between nodal flexibility and a behavioral factor related to psychosis-like experiences (F2) by the PW method (Figure 4). This association was significant with the majority of $\beta$ values implemented, that is, with $\beta=-$ .75 (fixed effect coefficient $=.013 \pm .0058,95 \%$ CI $[.0017 ; .0244], \mathrm{p}=.024, \mathrm{t}-\mathrm{stat}=2.3)$, with $\beta=-.25$ (fixed effect coefficient $=.016 \pm .0060,95 \%$ CI $[.0038 ; .0276], p=.009$, t-stat $=2.6$ ), with $\beta=0$ (fixed effect coefficient $=.018 \pm .0070,95 \%$ CI $[.0046 ; .0319], \mathrm{p}=.009, \mathrm{t}-$ 
Probabilistically Weighted Multilayer Networks disclose the link between default mode network instability and psychosis-like experiences in healthy adults

stat $=2.6$ ), and with $\beta=+.75$ (fixed effect coefficient $=.019 \pm .0062,95 \%$ CI $[.0070 ; .0314]$, $\mathrm{p}=.002$, t-stat $=3.1)$. Moreover, significant, negative associations were detected between nodal recruitment and the same behavioral factor F2 (Figure 5) with $\beta=-.75$ (fixed effect coefficient $=-.013 \pm .0067,95 \%$ CI $[-.0266 ;-.0003], \mathrm{p}=.045, \mathrm{t}-\mathrm{stat}=-2.0)$, with $\beta=-.75$ $($ fixed effect coefficient $=-.019 \pm .0051,95 \%$ CI $[-.0277 ;-.0078], \mathrm{p}=.0004$, t-stat $=-3.5)$, and with $\beta=-.75$ (fixed effect coefficient $=-.013 \pm .0067,95 \% \mathrm{CI}[-.0266 ;-.0007], \mathrm{p}=.039$, $\mathrm{t}$-stat $=-2.1)$. No significant results were observed regarding promiscuity or integrity.

As shown in Figures 4 and 5, these associations were strongest in regions of the default mode network (DMN), with the greatest effect sizes associated to regions in medial prefrontal cortex and posterior cingulate cortex. Interestingly, larger values of $\beta$ identified a larger

DMN associated with behavior. In other words, decreased probabilities of intra-nodal connections increased the likelihood of a larger group of regions to be progressively involved in the brain-behavior associations with respect to node flexibility and recruitment.

\section{DISCUSSION}

The primary aim of this study was to test the performance of the probabilistically weighted (PW) method for the detection of cross-layer communities. We implemented this method both on synthetic, perturbated time series of a network and on real data from resting-state fMRI. When applied on synthetic data, the PW method outperformed the standard $\omega=1$ method and was less affected by increasing noise levels in the time series. The better performance of the PW method was confirmed using two different indices of similarity in the modular structure, namely the adjusted mutual information index and the Rand coefficient and using modular structures with increasing number of modules. We deduce that these results are the direct consequence of a reduced - or practically absent - bias of selection with respect to the strength of cross-layer intra-nodal connections in the PW method. When 
Probabilistically Weighted Multilayer Networks disclose the link between default mode network instability and psychosis-like experiences in healthy adults

applied to real data, the PW method was able to identify significant associations of two dynamic metrics, namely flexibility and recruitment, with behavioral measures. Instead, the standard $\omega=1$ method did not identify any significant brain-behavior association. Therefore, we postulate that probabilistically weighted multilayer networks may be a desirable implementation for future studies and should replace the standard, biased $\omega=1$ method. While the results from simulated data are quite explicatory by themselves, further discussion is deserved for the results on real resting-state fMRI data.

Flexibility indicates how frequently a node changes module allegiance over time over time. Instead, recruitment indicates the probability of a node to belong to the same community as other nodes from its own system. Thus, our results indicate that high levels of psychosis-like experiences (F2) in healthy adults correspond to a decreased stability over time of nodes within the DMN. These findings are in line with the role of the fragmentation of the DMN in the predisposition toward psychopathology involving psychosis and dysfunctional behavior ${ }^{33,34,56,57}$. Moreover, higher flexibility and less stable modular organization have already been shown in patients with schizophrenia ${ }^{10,13}$, whereas higher switching has been associated with impaired sleep and decreased behavioral performances ${ }^{5,58}$. In this framework, our findings also fit with default mode-specific network aberrancies observed in patients with psychotic disorders $^{32,36,40,41}$, which also have genetic underpinnings ${ }^{59}$. We contribute to this framework by showing for the first time an association between nodal instability within the DMN (especially in medial prefrontal and in posterior cingulate cortices) and psychosis-like experiences in a sample of healthy individuals. We remark that these findings were possible due to the implementation of the PW method here presented. Considering that an aberrant degree of psychosis-like experiences may imply difficulties in social interactions ${ }^{60}$ and eventually impaired social functioning ${ }^{61}$, future studies 
Probabilistically Weighted Multilayer Networks disclose the link between default mode network instability and psychosis-like experiences in healthy adults

are needed to assess how the decreased stability of self-networks (DMN) may predispose toward clinical self-disturbances in healthy populations ${ }^{42-44,61}$.

When constructing and analyzing brain activity and functional connectivity, ground truths are rare and unstable. However, advances in the understanding of dynamic brain networks point toward the existence of multi-scale entities in the brain architecture ${ }^{1-5,10-13,16}$. These advances followed recent developments in network science ${ }^{62-65}$, and generally indicate the necessity to implement unbiased methods for detecting communities ${ }^{66-72}$, possibly controlling for multiple stochasticity in the community organization, like in multi-resolution approaches ${ }^{32,73,74}$. In fact, the choice of the resolution parameter $\gamma$ is important since it regulates the number and size of communities in a network ${ }^{14-16}$. However, in multilayer networks, the weight of intra-nodal (cross-layer) links become crucial if we are interested in temporal features of the network (e.g., flexibility, recruitment, or promiscuity ${ }^{6-9}$ ). The implementation of PW multilayer networks allows an unbiased approach to the study of brain networks through multiple temporal resolutions and without a-priori guessing the strength of cross-layer connections. To note, although the method presented in this paper is intrinsically developed, and thus more suitable, for weighted networks, it can also be applied using binarized graphs since the intra-nodal weights can be directly transformed in a stochastic binary connection value.

Concluding, we presented a probabilistic, biologically-driven approach for the unbiased selection of cross-layer links in multilayer networks. More specifically, we introduced probabilistic links that can be investigated at multiple temporal resolutions, represented by multiple shape $(\beta)$ values in the distribution of intra-node connection weights, and that are biologically grounded since they are assigned based on the coherence across time windows. Our method allows to investigate multiple temporal resolutions in multilayer networks, paralleling the actual unbiased approach proposed for spatial resolution $(\gamma)$. The higher 
bioRxiv preprint doi: https://doi.org/10.1101/2021.05.17.444398; this version posted May 17, 2021. The copyright holder for this preprint (which

was not certified by peer review) is the author/funder, who has granted bioRxiv a license to display the preprint in perpetuity. It is made available under aCC-BY-NC-ND 4.0 International license.

Probabilistically Weighted Multilayer Networks disclose the link between default mode network instability and psychosis-like experiences in healthy adults

performance achieved by the PW method, together with the significant associations with psychotic-like experiences detected, testifies the usefulness of our unbiased approach towards the study of dynamic brain functioning as well as its behavioral and cognitive correlates.

\section{ACKNOWLEDGMENTS}

This work was supported by the "Departments of Excellence 2018-2022" initiative of the Italian Ministry of Education, Research, and University for the Department of Neuroscience, Imaging, and Clinical Sciences (DNISC) of the University of Chieti -Pescara.

We thank Richard F Betzel for his constructive comments on an initial draft of the work.

\section{DATA AVAILABILITY}

The code used for implementing the probabilistically weighted procedure will be made available on open platforms after publication.

\section{COMPETING INTEREST STATEMENT}

The authors have no competing interest to declare. 

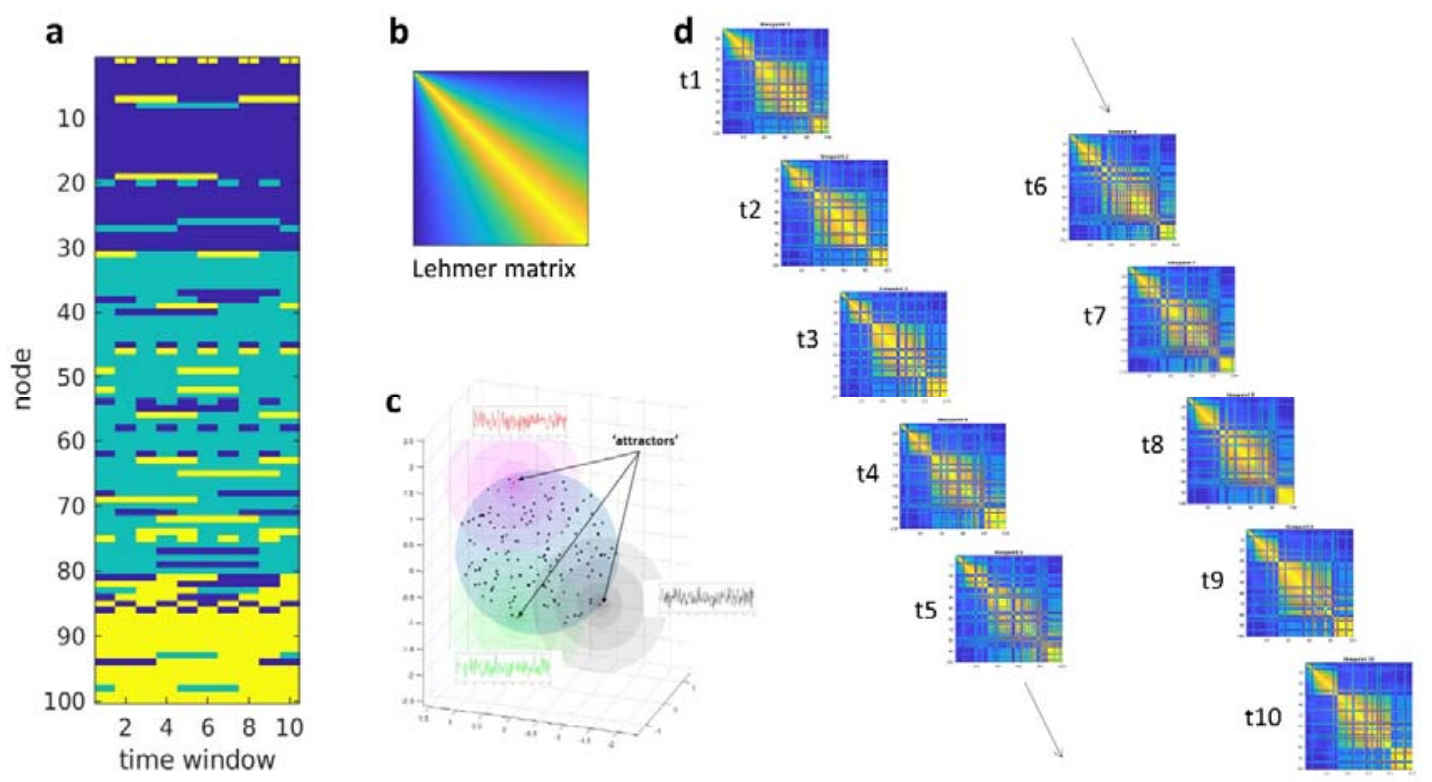

\section{FIGURES}

Figure 1. Synthetic multilayer networks. (A) The matrix of synthetic node communities was generated for ten consecutive timepoints. To note, the communities were different at each cycle. (B) A Lehmer matrix was initially simulated. Values inside the matrix range from 0 to 1 to simulate functional connectivity among brain regions. A time series was generated for each of the voxels within each of the 100 nodes. Each voxel was affected by unstructured and structured noise. (C. While the unstructured noise was a random time series generated independently for each voxel, the structured noise was node-specific. The subfigure depicts an example of "noisy attractors" which differentially influenced node's voxels depending on the distance. (D) For each module, nodes' time series were selectively time-shifted to create modular structures. After the procedure, at each cycle, a series of 100 timeseries arranged with the desired modular structure and with controlled signal-tonoise ratio was produced. These time series were used as input for the calculation of functional connectivity matrices. The figure illustrates the three-modules structure, but the same procedure was used for the five-modules structure. 
Probabilistically Weighted Multilayer Networks disclose the link between default mode network instability and psychosis-like experiences in healthy adults
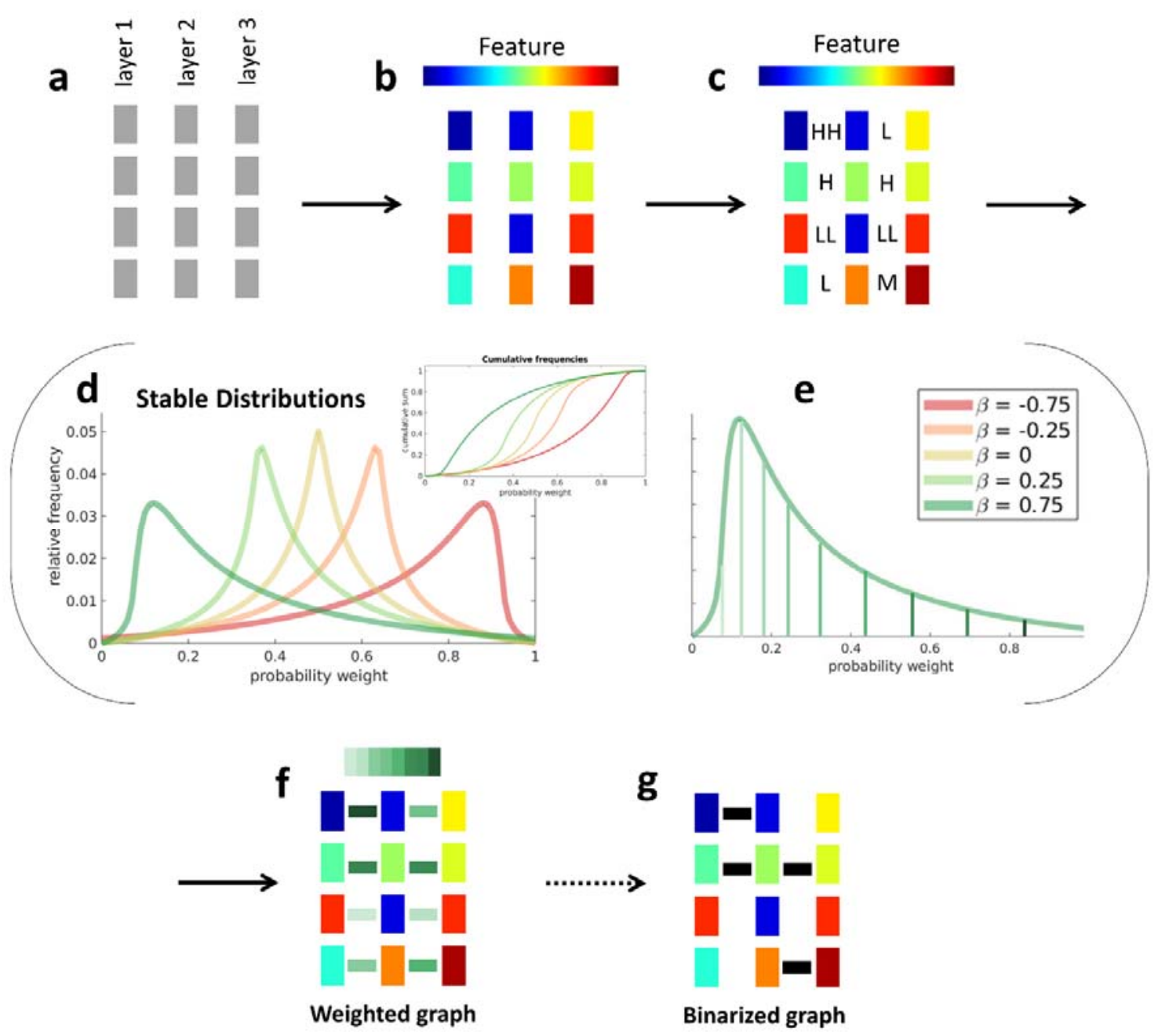

Figure 2. Toy example with 4 nodes and 3 layers (A), illustrating the procedure for estimating probabilistically weighted cross-layer links. Temporal (biological) features (e.g., frequencies distributions) are extracted for each node, in each layer (B). The similarity of temporal features is calculated for each adjacent layer (C) using coherence, producing a N x L-1 matrix, where $\mathrm{N}$ is the number of nodes and $\mathrm{L}$ is the number of layers ( $\mathrm{HH}=$ very high similarity; $\mathrm{LL}=$ very low similarity). Concurrently, a vector of $\mathrm{N}$ x L-1 probability weights, $\mathrm{W}$, is obtained from a sample distribution for each value of $\beta$ considered in the probabilistic analysis (D). For example, with beta $=0.75$, the majority of links will have a value between 0.1 and 0.2 (E). However, high similarities will be associated with higher values. Then, weights are assigned according to both the values in $\mathrm{W}$ and the signal similarity over time (coherence), so that cross-layer links connecting nodes with high self- 
bioRxiv preprint doi: https://doi.org/10.1101/2021.05.17.444398; this version posted May 17, 2021. The copyright holder for this preprint (which

was not certified by peer review) is the author/funder, who has granted bioRxiv a license to display the preprint in perpetuity. It is made available under aCC-BY-NC-ND 4.0 International license.

Probabilistically Weighted Multilayer Networks disclose the link between default mode network instability and psychosis-like experiences in healthy adults

similarity over time are higher $(\mathrm{F})$. Weights can be eventually used as probabilities for the linkage to occur in binary networks $(\mathrm{G})$. 
bioRxiv preprint doi: https://doi.org/10.1101/2021.05.17.444398; this version posted May 17, 2021. The copyright holder for this preprint (which was not certified by peer review) is the author/funder, who has granted bioRxiv a license to display the preprint in perpetuity. It is made available under aCC-BY-NC-ND 4.0 International license.

Probabilistically Weighted Multilayer Networks disclose the link between default mode network instability and psychosis-like experiences in healthy adults

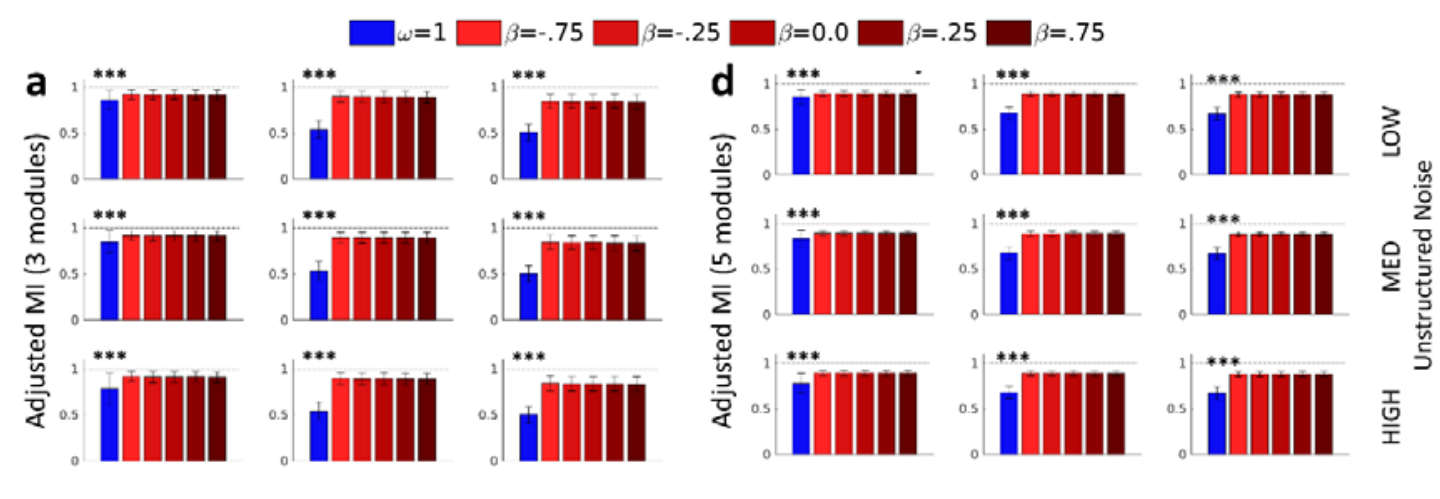

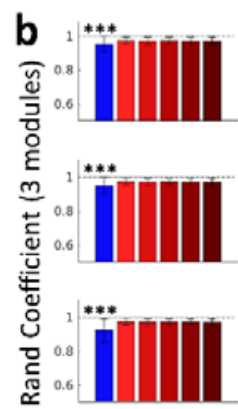

LoW

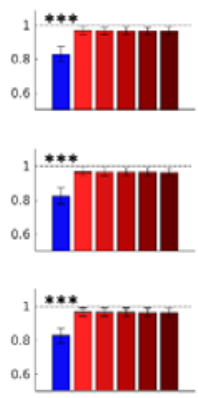

MED Structured Noise

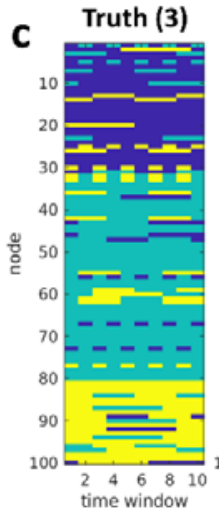

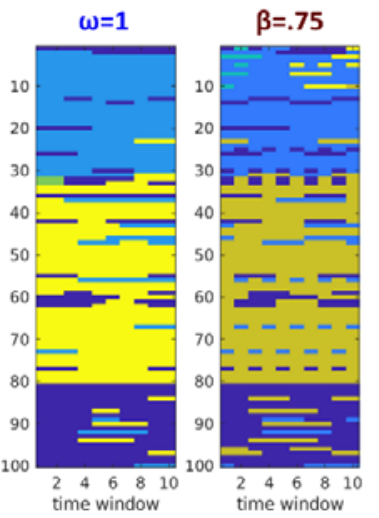

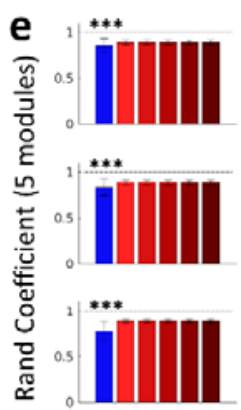

LOW

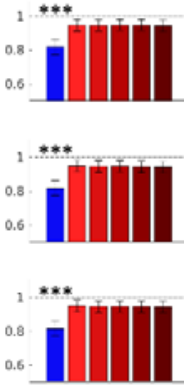

$\mathrm{HIGH}$

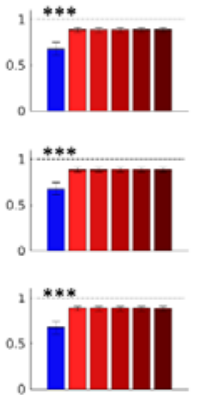

MED Structured Noise

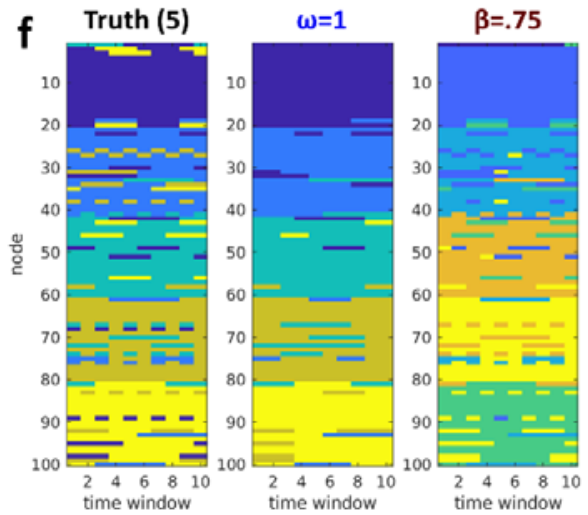

Figure 3. Results from simulated data. The PW method (red) outperformed the standard $\omega=1$

method (blue) considering adjusted mutual information (MI) indices as well as Rand coefficients. The result was consistent with both a three-modules $(\mathrm{A}, \mathrm{B}, \mathrm{C})$ and a five-modules $(\mathrm{D}, \mathrm{E}, \mathrm{F})$ network structure. Examples of differential community detection (for medium noise levels) are shown in (C) and (F). It is possible to appreciate that the standard method does not efficiently detect oscillator regions and may thus be eventually "confused" by flexible nodes. 
bioRxiv preprint doi: https://doi.org/10.1101/2021.05.17.444398; this version posted May 17, 2021. The copyright holder for this preprint (which was not certified by peer review) is the author/funder, who has granted bioRxiv a license to display the preprint in perpetuity. It is made available under aCC-BY-NC-ND 4.0 International license.

Probabilistically Weighted Multilayer Networks disclose the link between default mode network instability and psychosis-like experiences in healthy adults
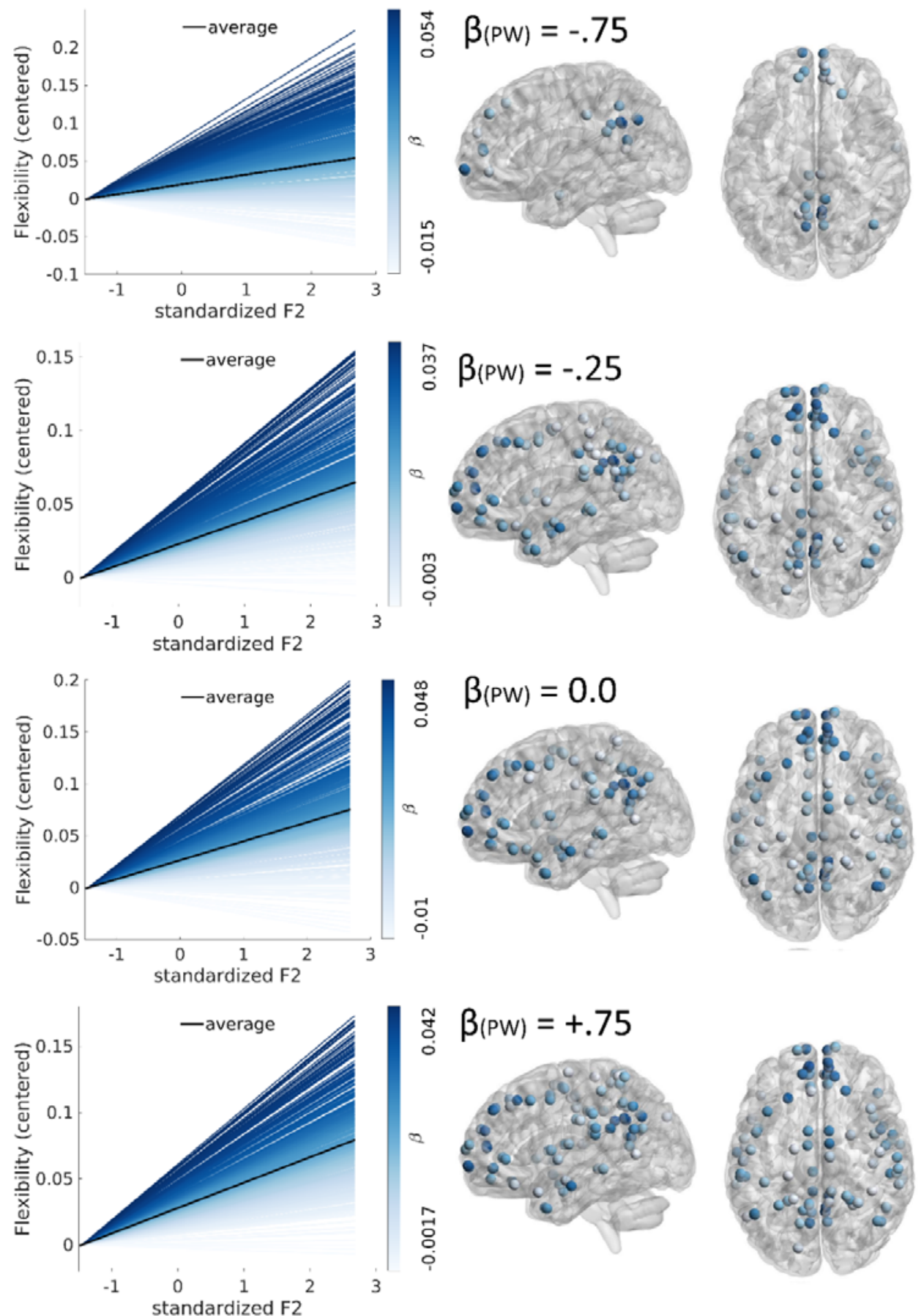

Figure 4. Significant associations between nodal flexibility and behavioral factor F2 (psychosis-

like experiences) caught by the PW method with increasing values of BETA. Each line in the ICE plot represents centered predictions for a node. Only nodes for which the slope was significantly higher than the average are represented in the brain topography. Colour intensity indicates the strength of the estimate. 
bioRxiv preprint doi: https://doi.org/10.1101/2021.05.17.444398; this version posted May 17, 2021. The copyright holder for this preprint (which

was not certified by peer review) is the author/funder, who has granted bioRxiv a license to display the preprint in perpetuity. It is made available under aCC-BY-NC-ND 4.0 International license.

Probabilistically Weighted Multilayer Networks disclose the link between default mode network instability and psychosis-like experiences in healthy adults
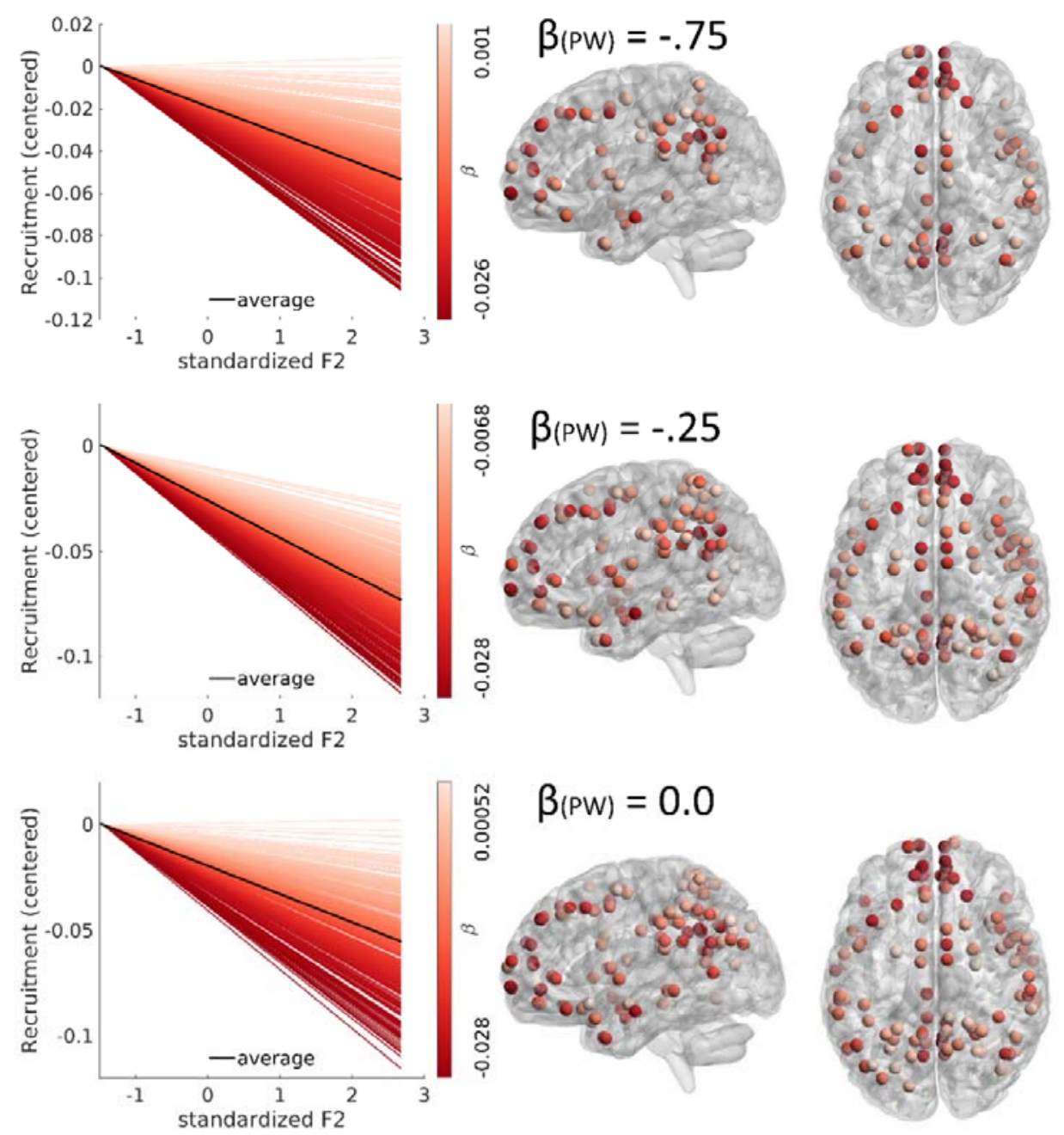

Figure 5. Significant associations between nodal recruitment and behavioral factor F2 (psychosis-

like experiences) caught by the PW method with increasing values of BETA. Only nodes for which the slope was significantly lower than the average are represented in the brain topography. Colour intensity indicates the strength of the estimate. 
Probabilistically Weighted Multilayer Networks disclose the link between default mode network instability and psychosis-like experiences in healthy adults

\section{REFERENCES}

1. Hutchinson, R.M., Womelsdorf, T., Allen, E.A., Bandettini, P.A., Calhoun, V.D., et al. Dynamic functional connectivity: Promises, issues, and interpretations. NeuroImage, 80, 360-372 (2013).

2. Boccaletti, S., Bianconi, G., Criado, R., et al. The structure and dynamics of multilayer networks. Phys Rep, 544(1), 1-122 (2014).

3. Kivela, M., Arenas, A., Barthelemy, M. et al. Multilayer Networks. Journal of Complex Networks, 2(3), 203-271 (2014).

4. De Domenico, M. Multilayer modelling and analysis of human brain networks. GigaScience, 6, 1-8 (2017).

5. Thompson, S.H., Falk, E.B., Vettel, J.M., Bassett, D.S. Network Approaches to Understand Individual Differences in Brain Connectivity: Opportunities for Personality Neuroscience. Personality Neuroscience, 1(e5), 1-12 (2018).

6. Bassett, D.S., Wymbs, N.F., Porter, M.A., Mucha, P.J., Carlson, J.M., Grafton, S.T. Dynamic reconfiguration of human brain networks during learning. PNAS, 108(18), 76417646 (2011).

7. Papadopoulos, L., Puckett, J.G., Daniels, K.E., Bassett, D.S. Evolution of network architecture in a granular material under compression. Phys Rev E, 94, 032908 (2016).

8. Pedersen, M., Zalesky, A., Omidvarnia, A., Jackson, G.D. Multilayer network switching rate predicts brain performance. PNAS, 115(52), 13376-13381 (2018).

9. Telesford, Q.K., Ashourvan, A., Wymbs, N.F., Grafton, S.T., Vettel, J.M., Bassett, D.S. Cohesive network reconfiguration accompanies extended training. Human Brain Mapping, 38(9), 4744-4759 (2017).

10. Braun, U., Schafer, A., Walter, H., Erk, S., Romanczuk-Seiferth, N, et al. Dynamic reconfiguration of frontal brain networks during executive cognition in humans. PNAS, 112(37), 11678-11683 (2015). 
Probabilistically Weighted Multilayer Networks disclose the link between default mode network instability and psychosis-like experiences in healthy adults

11. Telesford, Q.K., Lynall, M.-E., Vettel, J.M., Miller, M.B., Grafton, S.T., Bassett, D.S.

Detection of functional brain network reconfiguration during task-driven cognitive states.

NeuroImage, 142, 198-210 (2016).

12. Zheng, H., Li, F., Bo, Q., Li, X., Yao, L., et al. The dynamic characteristics of the anterior cingulate cortex in resting-state fMRI of patients with depression. J Affect Disord, 227, 391-397 (2018).

13. Gifford, G., Crossley, N., Kempton, M.J., Morgan, S., Dazzan, P., Young, J., McGuire, P. Resting state fMRI based multilayer network configuration in patients with schizophrenia. NeuroImage: Clinical, 25, 102169 (2020).

14. Lancichinetti, A., Fortunato, S. Community detection algorithms: A comparative analysis. Phys Rev E (2009).

15. Lancichinetti, A., Fortunato, S. Consensus clustering in complex networks. Sci Rep (2012).

16. Betzel, R.F., Bassett, D.S. Multi-scale brain networks. NeuroImage, 160, 73-83 (2017).

17. Bassett, D.S., Porter, M.A., Wymbs, N.F., Grafton, S.T., Carlson, J.M., Mucha, P.J. Robust detection of dynamic community structure in networks. Chaos: An Interdisciplinary Journal of Nonlinear Sciences, 23(1), 013142 (2013).

18. Gu, S., Pasqualetti, F., Cieslak, M., Telesford, Q.K., Alfred, B.Y., et al. Controllability of structural brain networks. Nature Communications, 6 (2015).

19. Betzel, R.F., Medaglia, J.D., Papadopoulos, L., Baum, G., Gur, R., et al. The modular organization of human anatomical networks: Accounting for the cost of wiring. Network Neuroscience, 1(1), 42-68 (2017).

20. Nicolini, C., Bifone, A. Modular structure of brain functional networks: breaking the resolution limit by surprise. Scientific reports 6 (2016).

21. Muldoon, S., Bassett, D.S. Network and multilayer network approaches to understanding human brain dynamics. Philosophy of Science, 83(5) (2016).

22. De Domenico, M., Solé-Ribalta, A., Cozzo, E., Kivela, M., Moreno, Y., et al. Mathematical formulation of multilayer networks. Phys Rev X, 3(4), 041022 (2013). 
Probabilistically Weighted Multilayer Networks disclose the link between default mode network instability and psychosis-like experiences in healthy adults

23. Chai, L., Mattar, M.G., Blank, I.A., Fedorenko, E., Bassett, D.S. Functional network dynamics of the language system. Cerebral Cortex, 26(11), 4148-4159 (2016).

24. Van De Ville, D., Britz, J., Michel, C.M. EEG microstate sequences in healthy humans at rest reveal scale-free dynamics. PNAS, 107, 181-79-18184 (2010).

25. De Pasquale, F., Della Penna, S., Sporns, O., Romani, G..L, Corbetta, M. A dynamic core network and global efficiency in the resting human brain. Cerebral Cortex, 26(10), 40154033 (2016).

26. Breakspear, M. Dynamic models of large-scale brain activity. Nature Neuroscience, 20, 340-352 (2017).

27. De Pasquale, F, Corbetta, M, Betti, V, Della Penna, S. Cortical cores in network dynamics. NeuroImage, 180, 370-382 (2018).

28. O’Neil, G.C., Bauer, M., Woolrich, M.W., Morris, PG., Barnes, G.R., Brookes, M.J. Dynamic recruitment of resting state sub-networks. NeuroImage, 115, 85-95 (2015).

29. Preti, M.G., Bolton, T.A., Van De Ville, D. The dynamic functional connectome: State-ofthe-art and perspectives. NeuroImage, 160, 41-54 (2016).

30. Spadone, S., Della Penna, S., Sestieri, C., Betti, V., Tosoni, A., et al. Dynamic reorganization of human resting-state networks during visuospatial attention. PNAS, 112(26), 8112-8117 (2018).

31. Wens, V., Bourguignon, M., Vander Ghinst, M., Mary, A., Marty, B., et al. Synchrony, metastability, dynamic integration, and competition in the spontaneous functional connectivity of the human brain. NeuroImage, 199, 313-324 (2019).

32. Di Plinio, S., Perrucci, M.G., Aleman, A., Ebisch, S.J.H. I am Me: Brain systems integrate and segregate to establish a multidimensional sense of self. NeuroImage, 205, 116284 (2020a).

33. Broyd, S.J., Demanuele, C., Debener, S., Helps, S.K, James, C.J., Sonuga-Barke, E.J.S. Default-mode brain dysfunction in mental disorders: A systematic review. Neuroscience and Biobehavioral Reviews, 33(3), 279-296 (2009). 
Probabilistically Weighted Multilayer Networks disclose the link between default mode network instability and psychosis-like experiences in healthy adults

34. Hua, J.P.Y., Karcher, N.R., Merrill, A.M., O’Brien, K.J., Straub, K.T., Trull, T.J., Kenrs, J.G. Psychosis risk is associated with decreased resting-state functional connectivity between the striatum and the default mode network. Cognitive, Affective, $\&$ Behavioral Neuroscience, 19, 998-1011 (2019).

35. Braun, U., Schafer, A., Bassett, D.S., Rausch, F., Schweiger, J.J., et al. Dynamic brain network reconfiguration as a potential schizophrenia genetic risk mechanism modulated by NMDA receptor function. PNAS, 113(44), 12568-12573 (2016).

36. Du, Y., Fryer, S.L., Fu, Z., Lin, D., Sui, J., et al. Dynamic functional connectivity impairments in early schizophrenia and clinical high-risk for psychosis. Brain Connectivity Dynamics, 180, 632-645 (2018).

37. Liu, X., Wu, X., Zhong, M., Huang, H., Weng, Y., et al. Dynamic Properties of Human Default Mode Network in Eyes-Closed and Eyes-Open. Brain Topography, 33, 720-732 (2020).

38. Bowyer, S.M. Coherence as a measure of the brain networks: Past and present. Neuropsychiatric Electrophysiology, 2(1) (2016).

39. Mohanty, R., Sethares, W.A., Nair, V.A., Prabhakaran, V. Rethinking measures of functional connectivity via feature extraction. Scientific Reports, 10(1298) (2020).

40. Ebisch, S.J.H., Aleman, A. The fragmented self: imbalance between intrinsic and extrinsic self-networks in psychotic disorders. The Lancet Psychiatry, 3(8), 784-790 (2016).

41. Humpston, C.S., Broome, M.R. Thinking, believing, and hallucinating self in schizophrenia. The Lancet Psychiatry, 7(7), 638-646 (2020).

42. McGrath, J.J., Saha, S., Al-Hamzawi, A., et al. Psychotic experiences in the general population: a cross-national analysis based on 31,261 respondents from 18 countries. JAMA Psychiatry, 72(7), 697-705 (2015).

43. Orr, J.M., Turner, J.A., Mittal, V.A. Widespread brain dysconnectivity associated with psychotic-like experiences in the general population. NeuroImage: Clinical, 4, 343-351 (2014). 
Probabilistically Weighted Multilayer Networks disclose the link between default mode network instability and psychosis-like experiences in healthy adults

44. Humpston, C.S. Perplexity and Meaning: Toward a Phenomenological "Core" of Psychotic Experiences. Schizophrenia Bulletin, 40(2), 240-243 (2014).

45. Joliot, M., Jobard, G., Naveau, M., Delcroix, N., Petit, L.,et al. AICHA: An atlas of intrinsic connectivity of homotopic areas. 254, 46-59 (2015).

46. Diedrichsen, J., Balster, J.H., Flavell, J., Ramnani, N. A probabilistic MR atlas of the human cerebellum. NeuroImage, 46(1), 39-46 (2009).

47. Di Plinio, S., Arnò, S., Perrucci, M.G., Ebisch, S.J.H. Environmental control and psychosis-relevant traits modulate the prospective sense of agency in non-clinical individuals. Consciousness and Cognition, 73, 102776 (2019).

48. Di Plinio, S., Arnò, S., Perrucci, M.G., Ebisch, S.J.H. The evolving sense of agency: Context recency and quality modulate the interaction between prospective and retrospective processes. Consciousness and Cognition, 80, 102903 (2020b).

49. Asai, T., Sugimori, E., Bando, N., Tanno, Y. The hierarchic structure in schizotypy and the five-factor model of personality. Psychiatry Research, 185(1-2), 78-83 (2011)

50. Gallagher, S., Trigg, D. Agency and Anxiety: Delusions of control and loss of control in Schizophrenia and Agoraphobia. Frontiers in Human Neuroscience, 10, 459 (2016).

51. Soto, C.J., John, O.P. Short and extra-short forms of the Big Five Inventory-2: The BFI-2-S and BFI-2-XS. J Res Pers, 68, 69-81 (2017).

52. Carleton, R.N., Norton, M.A., Asmundson, G.J.G. Fearing the unknown: A short version of the intolerance of uncertainty scale. J Anxiety Disord, 21, 105-17 (2007).

53. Raine, A. The SPQ: a scale for the assessment of schizotypal personality based on DSM-IIR criteria. Schizophrenia Bull, 17(4), 555-64 (1991).

54. Konings, M., et al. Validity and reliability of the CAPE: a self-report instrument for the measurement of psychotic experiences in the general population. Act Psychiat Scand, 114(1), 55-61 (2006).

55. Spielberger, C.D., et al. Manual for the State-Trait Anxiety Inventory. Consulting Psychologists Press (1983). 
Probabilistically Weighted Multilayer Networks disclose the link between default mode network instability and psychosis-like experiences in healthy adults

56. Fan, F., Tan, Y., Wang, Z., Yang, F., Fan, H., et al. Functional fractionation of default mode network in first episode schizophrenia. Schizophrenia Research, 210, 115-121 (2019).

57. Hu, M.-L., Zong, X.-F., Mann, J.J., Zheng, J.-J., Liao, Y.-H., et al. A Review of the Functional and Anatomical Default Mode Network in Schizophrenia. Neuroscience Bulletin, 33(1), 73-84 (2017).

58. Pedersen, M., Zalesky, A., Omidvarnia, A., Jackson, G.D. Multilayer network switching rate predicts brain performance. PNAS, 115(52), 13376-13381 (2018).

59. Scariati, E., Schaer, M., Richiardi, J., Schneider, M., Debbané, M., Van De Ville, D., Eliez, S. Identifying 22q11.2 Deletion Syndrome and Psychosis Using Resting-State Connectivity Patterns. Brain Topography, 27, 808-821 (2014).

60. De Bézenac, C.E., Sluming, V., O’Sullivan, N., Corcoran, R. Ambiguity between self and other: Individual differences in action attribution. Consciousness and Cognition, 35, 1-15 (2015).

61. Nelson, B., Thompson, A., Yung, A.R. Basic self-disturbance predicts psychosis onset in the ultra high risk for psychosis "prodromal” population. Schizophrenia Bulletin, 38, 12771287 (2012).

62. Newman, M.E. The structure and function of complex networks. SIAM Rev, 45(2), 167256 (2003).

63. Bullmore, E, Sporns, O. Complex brain networks: Graph theoretical analysis of structural and functional systems. Nat Rev Neurosci, 10(3), 186-188 (2009).

64. Rubinov, M., Sporns, O. Complex network measures of brain connectivity: uses and interpretations. NeuroImage, 52(3), 1050-1060 (2010).

65. Bassett, D.S., Sporns, O. Network Neuroscience. Nature Neuroscience, 20, 353-364 (2017).

66. Newman, M.E., Girvan, M. Finding and evaluating community structure in networks. Phys Rev E, 69(2), 026113 (2004). 
Probabilistically Weighted Multilayer Networks disclose the link between default mode network instability and psychosis-like experiences in healthy adults

67. Porter, M.A., Onnela, J., Mucha, P.J. Communities in networks. Not Amer Math Soc, 56(9), 1082-1097 (2009).

68. Sporns, O., Betzel, R.F. Modular brain networks. Ann Rev Psychol, 67, 613-640 (2016).

69. Fenn, D.J., Porter, M.A., McDonald, M., Williams, S., Johnson, N.F., Jones, N.S.

Dynamical clustering of exchange rates. Quant Financ, 12(10), 1493-1520 (2012).

70. Fortunato ,S. Community detection in graphs. Phys Rep, 486(3), 75-174 (2010).

71. Fortunato, S., Hric, D. Community detection in networks: A user guide. Physics Reports, 659(11), 1-44 (2016).

72. Lancichinetti, A., Radicchi, F., Ramasco, J.J., Fortunato, S. Finding statistically significant communities in networks. PLoS One, 6(4), e18961 (2011).

73. Malagurski, B., Liem, F., Oschwald, J., Mérillat, S., Jancke, L. Longitudinal brain network reconfiguration in healthy aging. Human Brain Mapping, 41(17), 4829-4845 (2020).

74. Rubinov, M., Ypma, R.J., Watson, C., Bullmore, E.T. Wiring cost and topological participation of the mouse brain connectome. PNAS, 112(32), 10032-10037 (2015). 